\title{
EVALUATING EMERGENCY RESPONSE MODELS OF RADIOLOGICAL DISPERSION IN COMPLEX TERRAIN
}

\author{
L. L. Dyer, J. H. Pascoe \\ ${ }^{1}$ Safety and Radiation Services, Australian Nuclear Science and Technology Organisation, Sydney, Australia
}

\begin{abstract}
Operational airborne releases of trace quantities of the radioactive noble gas Ar-41 from the HIFAR Nuclear Research Reactor located in Sydney, Australia are valuable for evaluating emergency response models incorporating radiological dispersion. The Australian Nuclear Science and Technology Organisation (ANSTO), where the reactor is located, has a network of meteorological stations and GR-150 environmental gamma dose detectors placed in complex terrain within a $5 \mathrm{~km}$ radius of the site. The current version of ANSTO's Emergency Response System feeds real-time meteorological data from this network into the wind field model NUATMOS, which is then subsequently used, together with known source emissions of Ar-41 from HIFAR, to drive the Lagrangian mesoscale atmospheric dispersion puff model RIMPUFF. Output from RIMPUFF is compared with data from the gamma dose monitoring network in order to assess the overall performance of the system.

An updated model combination LSMC/RIMPUFF has recently been evaluated using Ar-41 peak observations from the detector network during 2002-03 under a range of atmospheric stability conditions. Sensitivity tests of the new model version were performed by perturbing the gridded meteorological data within the model domain, which incorporates complex terrain. Results from statistical analyses of the model output are reported, assessing the suitability of LSMC/RIMPUFF to replace the current models in the Emergency Response System.

The LSMC/RIMPUFF evaluation results are compared with two previous evaluations carried out in 2004, of LSMC's predecessor LINCOM/RIMPUFF and the NUATMOS/RIMPUFF combination currently used at ANSTO. Results from these tests have been favourable for LSMC/RIMPUFF, which is consequently now likely to replace the older models within ANSTO's Emergency Response System. The decision support system ARGOS, which also incorporates the LSMC/RIMPUFF model, is currently being evaluated for potential use by a number of Organisations within Australia including ANSTO. Some results of this initial evaluation will also be included here.
\end{abstract}

Key words: model evaluation, atmospheric dispersion models, gamma monitoring system, emergency response system.

\section{INTRODUCTION}

The Australian Nuclear Science and Technology Organisation (ANSTO) operates several national facilities including Australia's current nuclear research reactor, OPAL, located at Lucas Heights, Sydney. OPAL replaced the HIFAR reactor in January, 2007. HIFAR was used for materials research, to produce radioactive material for medicine and industry, conduct cutting-edge neutron beam science and irradiate silicon for the high performance computer industry. As part of ANSTO's Environmental Management System, there is continuous monitoring of airborne emissions from ANSTO facilities and effective dose rates to the public are modelled using the release data along with concurrent meteorological measurements. In particular for this study, routine airborne release data from HIFAR of the radioactive noble gas Ar-41 provided a valuable tool for evaluating emergency response models of radiological dispersion.

ANSTO's emergency response system utilises an Environmental Radiological Atmospheric Impact Modelling System (ERAIMS) to model dispersion for a range of potential airborne release scenarios. Using real-time meteorological data, ERAIMS predicts and maps the affected areas and concentrations in air. This provides the capability to respond for any potential accident with airborne consequences at Lucas Heights (Clark et al., 2003b). A challenge for accurately modelling plume dispersion at Lucas Heights is the complex terrain of dissected plateaus and valleys that surrounds ANSTO. This terrain has a significant influence on the movement of airborne particles. Model evaluations and inter-comparisons are presented here with a discussion of the replacement of models within ANSTO's emergency response system. The incorporation of the decision support system ARGOS into ANSTO's emergency response system will also be discussed. ARGOS incorporates the model LSMC/RIMPUFF and is currently being evaluated Australia-wide for potential use at a number of emergency services organisations.

\section{METHODOLOGY}

\section{Meteorological and Environmental Gamma Monitoring System}

ANSTO has a network of three Meteorological stations and four GR-150 environmental gamma dose detector stations (see Figure 1). Meteorological data is collected at multiple heights from the $49 \mathrm{~m}$ tower on site and at single levels in surrounding areas of Shackles Estate (SE) and Boys Town (BT) stations. Meteorological statistics such as temperature, wind speed, wind direction and rainfall are collected every 15 minutes and used in the emergency operations centre. The gamma monitoring detection system was developed by Exploranium Canada (Grasty et al., 2001). The locations of these monitors include Lucas Heights ( $\mathrm{LH}, 0.82 \mathrm{~km}$ from HIFAR), Waste Services (WS, 0.73 $\mathrm{km})$ and the more distant stations at Barden Ridge $(\mathrm{BR}, 3.33 \mathrm{~km})$ and Boys Town $(\mathrm{BT}, 2.78 \mathrm{~km})$ denoted by undulating and complex terrain respectively (see Fig. 1). 


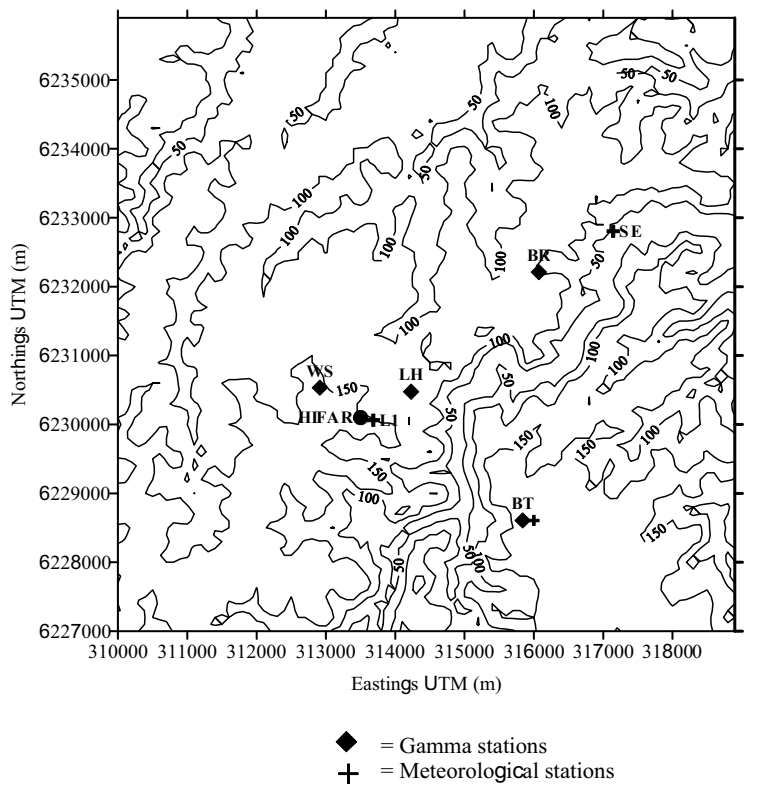

Figure 1. The Lucas Heights region showing locations of meteorological and environmental gamma monitoring stations with topographic features

\section{Atmospheric Dispersion Models}

The RIMPUFF (RIso Mesoscale PUFF) dispersion model (Mikkelsen et al., 1984) from Riso National Laboratories in Denmark has specifically been developed for nuclear applications. It is a Lagrangian mesoscale atmospheric dispersion puff model designed for calculating the concentration and doses resulting from the dispersion of airborne materials. The analyses presented in this paper involve the evaluation of RIMPUFF with The Local Scale Model Chain (LSMC; Thykier-Nielsen et al., 2004), the new version of the Riso dispersion modelling system and a submodule within MET-RODOS (Mikkelsen et al., 2002). A new feature of the LSMC/RIMPUFF model combination is that it integrates the wind field and dispersion calculations into one code whilst also using the meteorological data to calculate the wind field and parameters relating to the atmospheric stability. It has the advantage over past versions of incorporating modern micrometeorological scaling approaches into the vertical mixing and dispersion calculation. In particular, LSMC/RIMPUFF uses turbulent shear-stress, heat flux, Monin-Obukhov stability length and local mixing heights.

Statistical analyses and sensitivity analyses will be presented here to enable a comparison with previous evaluations of dispersion models. Previous model evaluations using the Ar-41 dataset include RIMPUFF with two different wind models: the wind field model LINCOM (LINearized COMputation), supplied by Riso (Troen, I. and A.F. de Bass, 1986) and also with the wind field model NUATMOS (New version of the ATMOS1 model; Davis et al., 1984), developed at Monash University (Ross et al., 1988). Both are diagnostic wind field models for complex terrain and LINCOM/RIMPUFF is the predecessor or LSMC/RIMPUFF. Early modelling studies indicated that LINCOM does not work well in the early to late morning transition period from stable nocturnal to daytime meteorological conditions (Clark et al., 2003a). Therefore, it was decided that NUATMOS with RIMPUFF would be best model combination for the emergency response system at ANSTO. More recent model evaluation results of LINCOM/RIMPUFF and NUATMOS/RIMPUFF are reported in a recent study by Williams et al. (2005) which serves as a useful benchmark for the evaluation presented here of LSMC/RIMPUFF. The model combinations with RIMPUFF are hereon in known as LINCOM, NUATMOS and LSMC.

\section{Case Studies}

The Ar-41 time series data used in this study were from November and December 2002 (summer) and in the winter months of June and July, 2003 when more stable atmospheric conditions were observed. The case studies at each of the monitoring stations covered all times during the day, thus the results were modelled under different atmospheric stability, wind speed, wind direction and dispersion conditions. To enable model evaluation during a range of atmospheric conditions, the results have been split into "unstable" and "stable" cases, based on Pasquill stability categories of the stability at the time of peak. "Unstable" is defined from very unstable (A) to neutral (D) and "Stable" is defined from stable (E) to very stable (F).

Characteristics of the terrain for each station that the plume passes over are mentioned in the results. LH and WS are within $1 \mathrm{~km}$ of the Ar-41 source with only gently sloping terrain nearby whereas the detector at BR lies further away and in a side gully leading to the main Woronora river valley. BT is on a plateau (or ridge) above this $100 \mathrm{~m}$ deep valley which might be expected to have a different influence on the dispersion processes. 


\section{Model Setup}

It is important to note the variations in model input files to enable an understanding of the differences found in the results. The source file is consistent with all models mentioned in this study. The meteorological data file however is the same for LINCOM and NUATMOS (due to RIMPUFF version 8 being used in both) but the new model LSMC uses a different formatted meteorological file. LSMC has the advantage of the stability calculated automatically from the meteorological data as mentioned earlier whereas the user defines the stability based on the Pasquill Stability method for LINCOM and NUATMOS. There are 5 different stability index modes (i.e. ISMODE) available within RIMPUFF. The LSMC and RIMPUFF User's Guide recommends to use ISMODE=1 (Pasquill Turner) only when data are insufficient for using ISMODE=5 (Similarity Approach) (Thykier-Nielsen et al., 2004). Due to the previous analyses of LINCOM and NUATMOS using the stability mode ISMODE=1, it was decided to use both ISMODE=1 and ISMODE $=5$ when evaluating LSMC. To compare the results here with previous analyses, the model input chosen includes only single level data for temperature, wind, net radiation and precipitation. Sensitivity tests were subsequently carried out using vertical profiles of temperature and wind from the main station to see if it improved the predictions.

\section{Model Evaluation Techniques}

Ratios of measured gamma data to model predictions have been calculated and used to obtain statistics. Common indicators of model performance are presented such as the fraction of predictions within factors 2 and 5 (FA2 and FA5) and the factor or excedance (FOEX), as defined in Mosca et al. (1998). The time difference between the model predicted peaks and the measured gamma peaks have also been calculated based on the 15 minute time interval.

\section{RESULTS}

\section{LSMC}

Statistics for LSMC have been calculated for the measured gamma data against model predicted peaks and shown in Figure 3. All results are calculated using data integrated over 15 minute intervals. The number of cases (i.e. peaks) studied are: for LH stable conditions is 23 and unstable 15, BT stable is 21 and unstable 11, WS unstable is 5 and BR stable is 4 . The station LH with near-flat terrain show significantly better results for the Pasquill stability mode ISMODE $=1$ than the Similarity Approach mode ISMODE $=5$ for stable and unstable cases for FA2 $(74 \%, 53 \%$ and $30 \%, 20 \%)$, FA5 $(100 \%, 80 \%$ and $91 \%, 80 \%)$, FOEX $(-24 \%,-23 \%$ and $-37 \%,-50 \%)$ and similar results between the modes for arrival times $(70 \%, 80 \%$ and $65 \%, 87 \%)$. It was suggested by the model developers to use ISMODE=5 for unstable cases to get a narrower and better simulated plume however our results here do not reflect this, ISMDODE $=1$ is best for unstable cases. Looking at how the modes perform under different stabilities, both ISMODE $=1$ and ISMODE $=5$ show better results under stable conditions for FA2 and FA5 but less well for arrival times. All stability modes here found the model under-predicting the peaks, particularly for ISMODE $=5$. Overall for the station LH, LSMC using ISMODE=1 under stable conditions give the best results when compared to the measurements.

Station WS also with near-flat terrain consists of 5 unstable cases only. Under those unstable conditions, ISMODE $=1$ performed much better for FA2 $(40 \%, 0 \%)$ and FOEX $(10 \%,-50 \%)$, the same for FA5 $(80 \%, 80 \%)$ but not quite as good as ISMODE $=5$ for arrival times $(60 \%, 100 \%)$. A larger number of cases would be more beneficial to explore the differences between the two stability modes and also under different atmospheric conditions. The station BR only consists of 4 stable cases where LSMC struggles to predict in the undulating terrain. There were poor results for ISMODE $=1$ and ISMODE $=5$ for FA2 $(0 \%, 0 \%)$, FA5 $(25 \%, 0 \%)$ and FOEX $(-50 \%,-50 \%)$ with more reasonable results for arrival times $(75 \%, 50 \%)$. All cases were under-predicted by the model with ISMODE $=1$ performing the better of the two modes overall for the stable conditions. Similarly to station WS, there needs to be a larger sample size to obtain reliable results.

Comparing the stability modes used for the station BT defined by complex terrain, ISMODE $=1$ produces significantly better results than ISMODE $=5$ for all tests; FA2 $(57 \%, 64 \%$ and $33 \%, 18 \%)$, FA5 $(90 \%, 82 \%$ and $90 \%$, $64 \%)$, FOEX $(-7 \%,-32 \%$ and $-50 \%,-50 \%)$ and arrival times $(86 \%, 73 \%$ and $76 \%, 73 \%)$. Looking at ISMODE $=1$ runs specifically, it performs best for stable cases than unstable cases for all tests except FA2 where the unstable case is slightly better. For ISMODE=5, the stable cases had either the same or slightly better results than the unstable cases for all tests. All stability modes here found the model under-predicting the peaks, particularly for ISMODE=5.

The results from this report's evaluation show that LSMC/RIMPUFF with stability mode ISMODE=1 performs either the same or significantly better than with ISMODE $=5$ under all stability conditions. This is true for all tests at all stations with only some exceptions for arrival times under unstable conditions where ISMODE $=5$ performs better at stations LH and WS. Further to those results, it can be concluded that LSMC using ISMODE=1 performs best under stable conditions for stations LH and BT where the greatest sample size exists. 

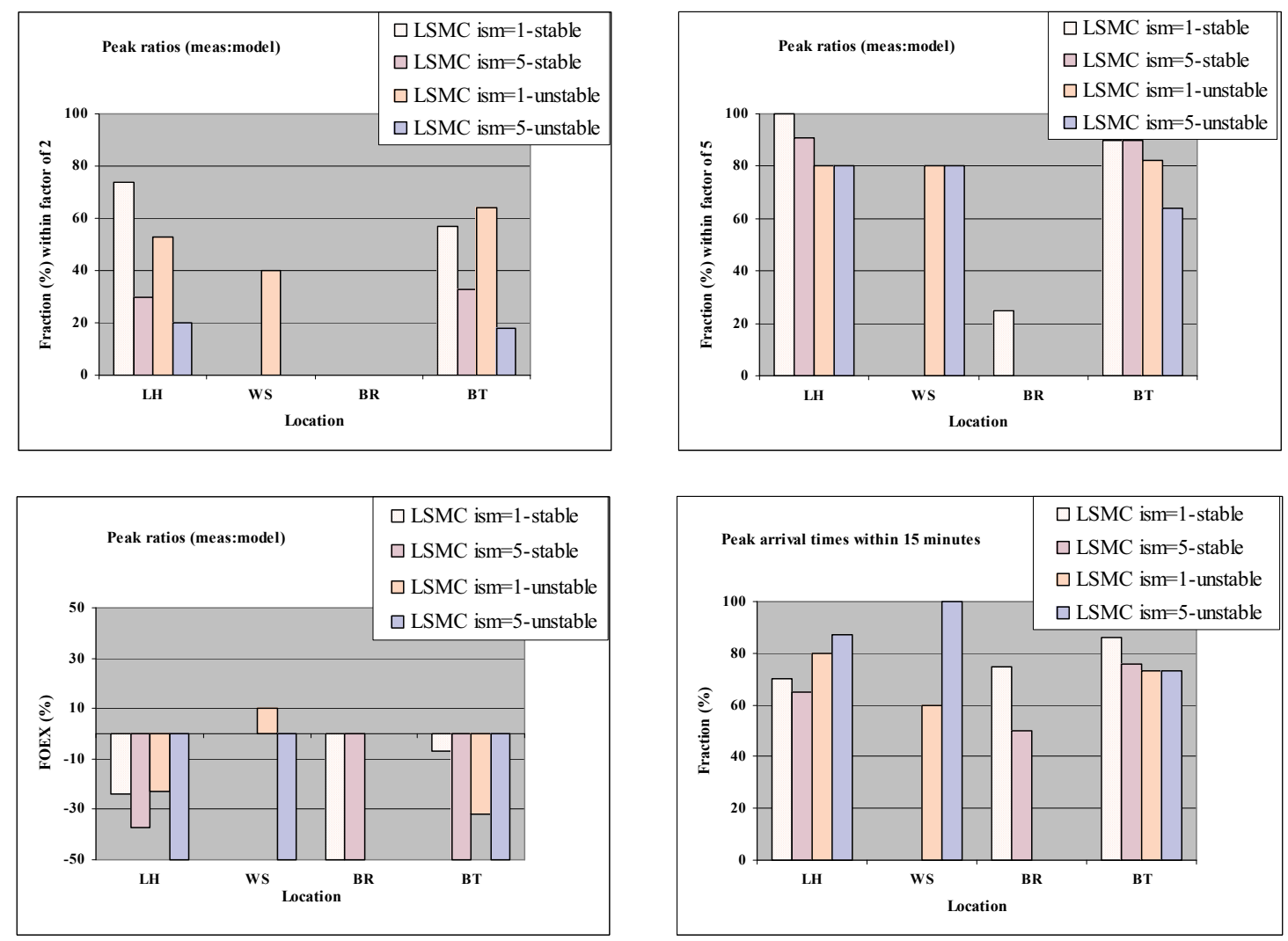

Figure 2. Summary statistics of the model LSMC/RIMPUFF against observations.

Further runs were carried out using meteorological input data such as vertical temperature and wind profiles. In particular the local station included the following: temperature at $2 \mathrm{~m}, 10 \mathrm{~m}$ and $49 \mathrm{~m}$, wind speed, direction, flux at $10 \mathrm{~m}$ and $49 \mathrm{~m}$, net radiation and precipitation. Unfortunately the LSMC results were not satisfactory and not even as good as LINCOM so further analyses were carried out. A number of cases were tested with different meteorological input files using only surface data at LH, with and without temperature data (for the nearby stations) and also leaving out the nearby stations altogether. All cases affected the results in a number of ways but no obvious conclusions were evident. Further analyses are needed for the vertical data to be incorporated into LSMC.

\section{Comparing LSMC with LINCOM and NUATMOS}

A dispersion model inter-comparison was carried out between LSMC and LINCOM and also between LSMC and NUATMOS, all using the Pasquill mode ISMODE=1. A summary of the outcomes will be given here although the data and graphs are not shown due to limited space. LSMC and LINCOM have very similar results for the station LH under all stabilities with LSMC performing slightly better than LINCOM under stable conditions and LINCOM performing better under unstable conditions. Williams et al. (2005) showed that NUATMOS was significantly better than LINCOM for the station BT with complex terrain and similarly here, LSMC is also significantly better than LINCOM at BT for all statistics under all stabilities. The most significant difference between the two model combinations for station WS is LSMC performing better with FA2 of 40\% compared to LINCOM's FA2 of $20 \%$. Whereas the station BR with undulating terrain, has LSMC not performing well for its stable cases with FA2 of $0 \%$ and LINCOM also poor with FA2 of $40 \%$. Even though at this station the peaks are arriving generally on time for LSMC, all values are being under-predicted. It should also be noted that LSMC performs best for arrival times at all stations.

The results of LSMC and NUATMOS for the flat terrain station LH are very good and similar for all statistics. LSMC performs slightly better than NUATMOS under stable conditions although it under-predicts slightly more than NUATMOS. Under unstable conditions, NUATMOS is slightly better except for arrival times. Both models have satisfactory results for the complex terrain station BT with the opposite pattern to the station LH where NUATMOS is slightly better for FA2 than LSMC for stable conditions however LSMC is better for unstable conditions. It is interesting that under unstable conditions at BT NUATMOS over-predicts whereas LSMC under-predicts. For the smaller sample sizes of station WS and BR, NUATMOS either performs the same or better than LSMC except for arrival times for BR, with very poor results for BR from both models. Once again LSMC performs best for arrival times for all stations. 


\section{DISCUSSION AND CONCLUSIONS}

A model evaluation of LSMC has been presented using Ar-41 peak observations from a network of detectors around ANSTO under a range of atmospheric stability conditions. Spatial plots, combined with peak ratios were found to be a very useful combination in these model analyses to determine how the model predicts the plume in the complex terrain around Lucas Heights.

Overall LSMC performs similarly or better than LINCOM and NUATMOS for the near flat stations LH and WS under all stability conditions. The one exception would be LSMC for unstable conditions where the model is only slightly behind the others in performance. All of the models evaluated here do not perform well for the station BR with undulating terrain and small sample size, especially LSMC failing to have any cases within a factor of 2 . The complex terrain towards station BT has proven difficult for LINCOM however the new version LSMC is much more satisfactory and results are more inline with the predictions of NUATMOS.

Preliminary evaluations of the decision support system ARGOS have included rating the performance of all aspects of the system including the: GUI; efficiency overall; databases; GIS tools; flexibility and ease of use; and reliability. Initial cases were tested that were relevant for ANSTO's applications and the system was overall found to be a useful tool to be used in addition to ANSTO's current emergency response system. ARGOS provides a more regional model prediction, uses NWP for prognosis, has a user-friendly interface and incorporates model input, running and output in one system. A limitation is the poorer model resolution compared to using the model LSMC alone as tested in these analyses. Thus, ARGOS could not replace ANSTO's current system but can be an addition to it.

Currently NUATMOS and RIMPUFF is the wind and model combination incorporated into the emergency response system at ANSTO based on its performance in past evaluations. LSMC evaluation results in this paper give confidence that LSMC could be implemented into ANSTO's emergency response system and replace the much older model NUATMOS. Further investigations will be carried out to determine the ultimate model set-up for LSMC especially for the Lucas Heights region. The variance in performance seen in all the models evaluated here show that it is a challenging test for models to predict flow in complex terrain, during all times of the day. As new models are developed, the large datasets generated from our gamma monitoring network will be invaluable for further model evaluation studies.

\section{REFERENCES}

Clark, G.H, J. Crawford and G. Peady, 2003a: A Technical Manual for the Environmental Radiological Atmospheric Impact Modelling System (ERAIMS) - Version 1 (LINCOM wind module) - Revision 1. ANSTO/M-132.

Clark, G.H, J. Crawford and G. Peady, 2003b: A Technical Manual for the Environmental Radiological Atmospheric Impact Modelling System (ERAIMS) - Version 2 (NUATMOS wind module) - Revision 1. ANSTO/M133.

Davis, C.G, S.S. Brunker and J.P. Mutschlecner, 1984: Atmospheric transport models for complex terrain. Journal Climate and Applied Meteorology, 23(2), 235-238.

Grasty, R.L, J. Hovgaard and J.R. LaMarre, 2001: A fence line noble gas monitoring system for nuclear power plants. Radiation Protection Dosimetry, 95(3), 249-256.

Mikkelsen, T, S. Thykier-Nielsen, P. Astrup, S. Deme, J.H. Sorensen, A. Rasmussen, J. Pasler-Sauer, T. Schishtel, W. Raskob, R. Martens and L. Robertsson, 2002: Functionality specification for the Local Scale Model Chain LSMC in RODOS. Report No. RODOS(WG2)-TN(98)-03 available from Riso National Laboratories, Denmark.

Mikkelsen, T, S.E Larsen and S. Thykier-Nielsen, 1984: Description of the Riso Puff Diffusion Model. Nuclear Technology, 67, 55-65.

Mosca, S, G. Graziani, W. Klug, R. Bellasio and R. Bianconi, 1998: A statistical methodology for the evaluation of long-range dispersion models: an application to the ETEX exercise. Atmospheric Environment, 32(24), 4307-4324.

Ross, D.G, I.N. Smith, P.C. Manins and D.G. Fox, 1988: Diagnostic wind field modelling for complex terrain: Model development and testing. Journal of Applied Meteorology, 27(7), 785-796.

Thykier-Nielsen, S, P. Astrup, S. Deme and T. Mikkelsen, 2004: LSMC and RIMPUFF Input parameters User's Guide, Department of Wind Energy Riso National Laboratory, Denmark, August 2004.

Troen, I and A.F. de Bass, 1986: A spectral diagnostic model for wind flow simulation in complex terrain. Proc. Euro. Wind Energy Conf., Rome, Oct 7-9.

Williams, A, G. Clark, L. Dyer and R. Barton, 2005: Nuclear tools for characterising radiological dispersion in complex terrain: evaluation of regulatory and emergency response models. Int. J. of Environment and Pollution, 24, 1-4. 